\title{
Phase transitions in argon films
}

\author{
Peter Day, Mark Lysek, Marissa LaMadrid, and David Goodstein \\ Condensed Matter Physics 114-36, California Institute of Technology, Pasadena, California 91125
}

(Received 10 February 1992; revised manuscript received 15 January 1993)

\begin{abstract}
We present heat-capacity data detailing the evolution of the first six layers of argon adsorbed on graphite foam. The second and third layers have liquid-solid-gas triple points similar to the first layer. These layers exhibit a phase diagram consisting of two-dimensional solid, liquid, and gas phases on top of a solid film. Above the temperatures of the individual-layer triple points, the melting transition for each layer appears to be first order, and the first two layers show evidence of registry transitions prior to melting. For films of a total thickness of about four layers and up, the melting of each of the first three layers occurs at temperatures above the bulk triple point, as reported by Zhu and Dash [Phys. Rev. B 38, 11673 (1988)]. Our results confirm those of an ellipsometry study [H. S. Youn and G. B. Hess, Phys. Rev. Lett. 64, 918 (1990)] that found layering transitions above what were believed to be the layering critical-point temperatures. We observe heat-capacity peaks identified with these transitions and with melting transitions that join them with the low-temperature layering transitions. A phase diagram based on these data may represent the signature of a preroughening transition and a disordered flat phase in the bulk-crystal interface.
\end{abstract}

\section{INTRODUCTION}

In recent years the focus of adsorbed film studies has shifted from monolayer to multilayer films. Argon adsorbed on graphite has been studied by a variety of techniques including heat capacity, ${ }^{1-4}$ vapor pressure, ${ }^{5} \mathrm{x}-\mathrm{ray}^{6}$ and neutron diffraction, ${ }^{7-9}$ ellipsometry, ${ }^{10}$ and computer simulations. ${ }^{11,12}$ Apart from helium, argon is probably the most studied adsorbed film. We present here new thermodynamic data that contradict some earlier results while agreeing with others. We also show several new details of the multilayer phase diagram undetected in earlier experiments.

By studying films that are several layers thick, we hope to gain insight into the behavior of crystal surfaces. To accomplish this task, careful consideration must be given to the influence of the substrate on the film. The difference between an adsorbed film and a crystal surface is twofold. First, the film exists in the Van der Waals potential of the graphite substrate. This potential allows condensed matter to exist at a chemical potential below that of the bulk, as described approximately by the Frenkel-Halsey-Hill ${ }^{13}$ equation. Since the substrate potential falls off approximately as the inverse cube of the distance from the substrate, the first layer finds itself in a relatively large potential, and the pressure of the vapor in equilibrium with a monolayer film is only a few torr at 77 $\mathrm{K}$, where the bulk saturated vapor pressure is 194 torr. The pressure of a bilayer film is approximately 70 torr, and that of a trilayer film 130 torr. It is only at about five layers that the vapor pressure is within 20 torr of the bulk. The second influence of the substrate is due to the corrugation of the graphite surface. It is known from neutron diffraction ${ }^{7-9}$ and $\mathrm{x}$ - -ay $^{6}$ experiments that monolayer argon forms a two-dimensional solid at low temper- ature with a lattice spacing smaller than that of the $\sqrt{3} \times \sqrt{3}$ graphite lattice. This type of solid lattice is usually denoted as a high-density (HD) phase. Adsorbates have been shown to form commensurate phases (C), where there is exactly one adsorbate molecule for every three adsorption sites, and low-density (LD) phases, where the lattice spacing is larger than the commensurate spacing. In argon, no $\mathrm{C}$ or LD phases have been identified by previous studies. Most other monolayer films, however, have been shown to exhibit commensurate-incommensurate transitions driven either by varying temperature, causing the film to expand or contract at a different rate than the substrate, or by varying the total film thickness, causing the overlayers to exert more or less stress on the first layer.

Experimental $^{14}$ and theoretical ${ }^{15-19}$ studies have produced a standard picture of the growth of films adsorbed on attractive substrates. At low temperatures, a multilayer film grows by means of first-order layering transitions. As the vapor pressure above the film is raised, a layer condenses, resulting in a vertical step in a number versus pressure isotherm. The step corresponds to the unique pressure at which an $N$-layer film may coexist with an $N+1$-layer film. At higher temperatures, the layering transitions end in layering critical points at which the film no longer prefers to have an integer coverage. Past the critical point, regions of $N$ - and $N+1$-layer films can no longer be distinguished.

In a study of bilayer CO films, ${ }^{20}$ it was found that the second layer may form solid, liquid, and gas phases on top of an ordered first layer. Thus, the substrate potential may stabilize the same sort of two-dimensional behavior in the second layer as is found in monolayer films. In this study, we find that both the second and third layers in argon form two-dimensional solid, liquid, 
and gas phases. Below $T_{t}$, the bulk triple-point temperature, the completed lower layers are generally solid and can be viewed as a substrate on which the uppermost layer is adsorbed. At low temperatures, the layering transition is equivalent to the first-order transition between solid and gas phases in the top layer. Above the Nthlayer triple-point temperature, coexistence between the $N$ th-layer liquid and gas phases occurs. This coexistence region, which ends in the layering critical point, is between two disordered phases. Also, above the layer triple-point temperature, a melting transition between solid and fluid phases still exists at higher coverage, but it is less important for film growth because the phases are of nearly the same density.

It has been shown $n^{3,21-25}$ that the influence of the substrate may stabilize the solid phase of the first few layers, pushing their individual melting temperatures even above that of the bulk. This effect, termed substrate-induced freezing, ${ }^{26}$ has been shown in simulations to be affected by modulations in the substrate potential. However, even in simulations done on smooth substrates, ${ }^{11}$ the layers closest to the substrate disorder at higher temperatures than the overlayers. Our data confirm that the first three layers of argon melt above the bulk triple-point temperature for a sufficiently thick overlying film. Thus, substrate-induced freezing can produce layer-by-layer melting in thin films.

Solid-on-solid (SOS) models ${ }^{18,19}$ exhibit layering transitions, but cannot describe all the details of the experimental system. In a SOS model, for example, the incomplete $N+1$ st layer has only two phases, ordered and disordered, corresponding to condensed and uncondensed phases, respectively, in real films. It has been shown in theoretical treatments of SOS models that the layer critical transitions converge, in the limit of infinite film thickness, to the roughening transition in the bulk. ${ }^{17,18}$ Roughening occurs at a crystal surface when the free energy associated with a step goes to zero. ${ }^{27}$ At the roughening temperature, a crystal can no longer sustain facets, and its surface becomes rounded. Formally, at the roughening transition the width of the interface becomes infinite, but that is impossible in a multilayer film. However, the tendency of the crystal to remain flat below the roughening temperature survives in multilayer films in the form of first-order layering transitions.

Based on the model calculations, it was thought that the disordering of the topmost layer of a thick film or crystal, which is identified with the critical point of a layering transition in a SOS model, was enough to unlock the surface to the formation of arbitrarily many steps. Thus, the disordering of the surface layer would lead to roughening. However, recent calculations by den Nijs, ${ }^{28}$ using the restricted solid-on-solid (RSOS) model, have revealed a new surface phase transition called preroughening (PR) at which a crystal surface becomes disordered but its width remains finite. In the disordered flat (DOF) phase between PR and roughening, an antiferromagnetic ordering of steps is preferred, and the mean occupancy of the surface layer is $\frac{1}{2}$. The disorder referred to here is that of a lattice model, as opposed to the melting of a two-dimensional (2D) solid into a 2D fluid phase.
It is expected that if preroughening occurs, a crystal's surface will disorder but remain faceted. This behavior has not yet been observed in experiments. In this study we are concerned with the manifestations of preroughening in multilayer films. The ellipsometry results of Youn and $\mathrm{Hess}^{10}(\mathrm{YH})$ suggest that a phenomenon associated with PR may occur in argon on graphite. This study found new layering transitions for the fourth and higher layers at temperatures above those at which the layering transitions were thought to end and at which the uppermost layer was thought to disorder. ${ }^{3,29} \mathrm{YH}$ refer to these as reentrant layering transitions. YH suggest that the reentrant and low-temperature coexistence regions are joined by melting transitions that they do not observe, so the coexisting phases in the reentrant regions are identified as $N$ and $N+1$ solid layers covered with about one-half layer of disordered film. ${ }^{14}$ Conversely, earlier heat-capacity data by Zhu and Dash ${ }^{3}$ were interpreted to mean that only conventional roughening occurs in argon. These data lacked the resolution necessary to find the features associated with the reentrant layering that we report below. The interpretation of these data was also hampered by a lack of an understanding of the role of capillary condensation in this system that has since developed. We are able to confirm the reentrant layering transitions and observe the connecting transitions, thus bringing heat-capacity data into agreement with ellipsometry, and substantiating YH's interpretation. In addition, we show how the phenomena in layers four and above evolve from strikingly different phenomena in the layers closer to the substrate.

\section{EXPERIMENTAL DETAILS}

The experiment was performed using a homemade adiabatic scanning ratio calorimeter. ${ }^{30,31}$ The noise in the heat-capacity data is about $0.04 \%$ of the total sample cell heat capacity, and the reproducibility of data taken from different runs at the same coverage is better than $0.1 \%$. The substrate is Union Carbide Grafoam which has been prepared by outgassing at $1000^{\circ} \mathrm{C}$ until the pressure was below $10^{-6}$ torr, after an initial $1500^{\circ} \mathrm{C}$ heating in a chlorine atmosphere when it was manufactured. The adsorbate is $99.995 \%$ pure argon gas. Heat-capacity scans were taken at 53 coverages between one and six layers, from 63 to $90 \mathrm{~K}$. A comparison thermal mass was heated with a constant heat input, producing a scan rate of approximately $3 \mathrm{~K}$ per $2 \mathrm{~h}$. Individual data points were taken at every half second and averaged to produce about ten statistically independent points per degree. Pressures were simultaneously measured during the heat-capacity scans with a 1000-torr Barocel capacitive manometer that is linear to within $0.03 \%$ of full scale, allowing a precise determination of the number adsorbed in the film at all temperatures. Temperatures were measured with a platinum thermometer read by a resistance bridge. The thermometer was calibrated to the triple point of methane $(90.685 \mathrm{~K})$ and is believed to be sufficiently accurate around the argon triple point $(83.806 \mathrm{~K})$. The unit of coverage in this study is taken to be one layer per 220 $\mathrm{cm}^{3}$ at standard temperature and pressure (STP) of gas 
corresponding to the inflection point of the first step of a 77-K argon isotherm. The $\sqrt{3} \times \sqrt{3}$ commensurate coverage is $165 \mathrm{~cm}^{3}$ at STP.

The heat-capacity scans were performed in order of increasing coverage to keep the film on the adsorption branch of the capillary condensation hysteresis loop. ${ }^{32}$ Thus, we seek to minimize the amount of capillary condensation in the system. Between scans, the film was dosed from a calibrated volume at room temperature by cooling the cell. The films were then annealed at $90 \mathrm{~K}$ and recooled according to a power-law temperature versus time protocol ${ }^{33}$ that is believed to aid in the formation of uniform films.

It is important to keep in mind that for films of four layers and above, a substantial part of the total number of atoms adsorbed in the system are condensed in the form of capillary condensate. Any film thicknesses quoted are therefore only nominal, corresponding to $220 \mathrm{~cm}^{3}$ at STP of gas adsorbed per nominal layer (found from the inflection point of a 77-K isotherm). Our simultaneous measurement of the vapor pressure above the film, however, permits us largely to overcome the problem of capillary condensation by citing our results in terms of chemical potential. The experimental procedure has been presented in detail elsewhere. ${ }^{30-32}$

\section{RESULTS}

In Sec. III A we report a vapor pressure isotherm that was used to investigate the behavior of capillary condensation in this system. In the rest of the paper, we will discuss the results of heat-capacity scans with accompanying pressure measurement showing the behavior of films of between one and six complete layers. In Secs. III B and III C, we describe behavior in the first three layers that is characteristic of two-dimensional matter. In Sec. III D, we describe behavior in films of four or more layers that we believe is characteristic of a crystal interface.

\section{A. Isotherm}

Figure 1 shows a 77-K isotherm executed by adsorbing and then withdrawing gas to map out the capillary condensation hysteresis curve. The isotherm is plotted as number adsorbed versus $\left(\mu_{0}-\mu\right)^{(-1 / 3)}$ to demonstrate the effect of capillary condensation on the system. According to the Frenkel-Halsey-Hill model, the isotherm should approximate a straight line (the dashed line shown) but with steps at even intervals indicating layer condensation. When atoms start to condense in pores and cracks, the adsorption isotherm curves sharply upward from the straight line. Once capillary condensation has been nucleated in this system, the number adsorbed in pores is expected to vary approximately as the number in the film to the sixth power. ${ }^{34}$ By fitting the isotherm in Fig. 1, we find that the deviation from the straight line approximately equals the film thickness to the $5 \pm 1$ power. By considering the spacing of the layer condensation steps, we find that the maximum film thickness attainable by putting reasonable amounts of gas into the sample cell is about six layers. In fact, we have made measurements of heat-capacity signals identified with the sixth film layer when the equivalent of about twenty layers of gas were adsorbed in the cell. An upper limit of six layers was also reported by a recent neutron-diffraction study that integrated diffraction peaks to estimate the number adsorbed with the bulk lattice spacing. The hysteretic behavior in Fig. 1 is virtually identical to that reported in methane on graphite. ${ }^{32}$ We see that along the desorption (low-pressure) branch of the curve, capillary condensation may coexist with a film as thin as 1.1 layers. As previously noted, we do not see a heat-capacity signature of melting of capillary condensate along the adsorption branch until about 3.5 layers are adsorbed. This too was observed in the case of methane on the same substrate. For argon as for methane on this substate, capillary condensate is a state of matter that coexists with ad-

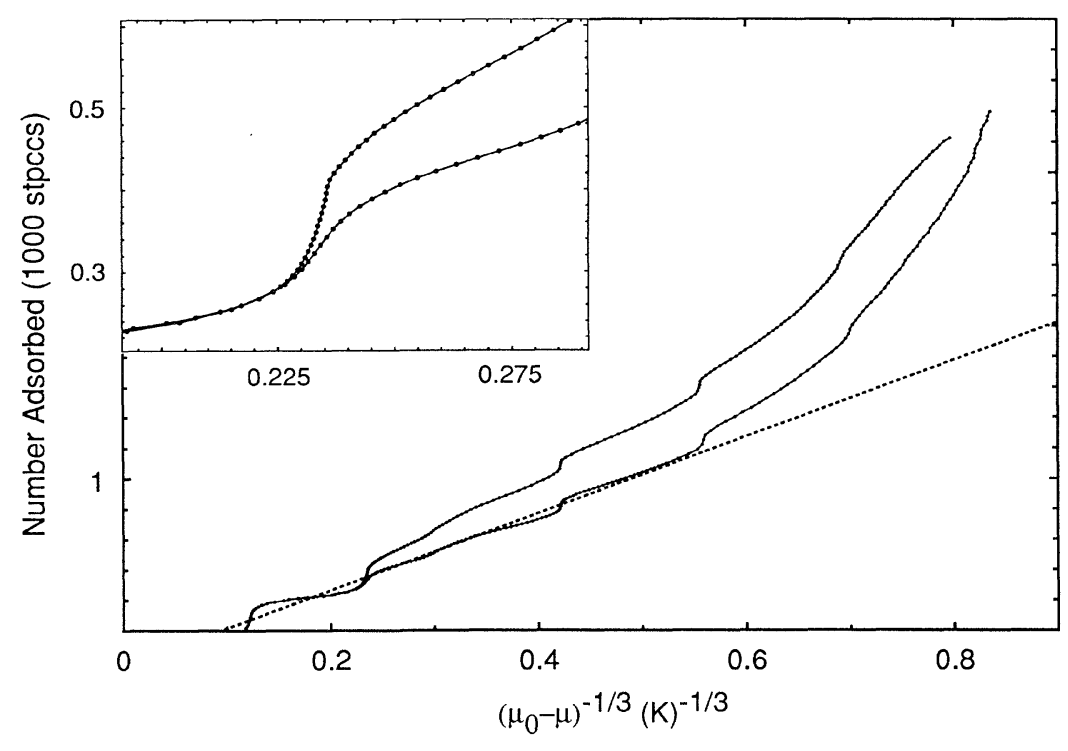

FIG. 1. Adsorption and desorption branches of a $77-\mathrm{K}$ isotherm. The inset shows the two curves joining at low coverage. The line is a least-squares fit to the low-coverage data. 
sorbed film without interacting significantly with it. Once the effects of capillary condensation are established, as we have done here, it becomes possible to study the behavior of uniform films up to about six layers thick.

\section{B. Low-temperature phase diagrams}

Figure 2 shows the low-temperature region of several heat-capacity scans between 1.00 and 4.95 layers. The heat capacities in this figure and those following are uncorrected for desorption. Scans $A-K$ showed no sign of a peak near the bulk triple-point temperature $T_{t}$ indicating the nucleation of capillary condensation. Thus we can take the number in the film for these scans to be approximately equal to the total number in the system minus the number in the vapor phase. As the temperature of the film increases, a substantial amount of film desorbs from the surface and enters the vapor. For the highest coverage scans, this amount is about 1.2 equivalent layers at $90 \mathrm{~K}$. Although this loss of particles from the film could, in theory, result in hysteresis, i.e., in the system leaving the adsorption branch on the next scan, we have found that the deviation is small by comparing pressures at $77 \mathrm{~K}$ from each scan to a $77-\mathrm{K}$ isotherm in which the film was formed by adsorption at constant temperature.

The scans in Fig. 2 show three groupings of peaks that we attribute to phenomena in the second (scans $A-F$ ), third (scans $G-L$ ), and fourth (scans $M-O$ ) film layers.

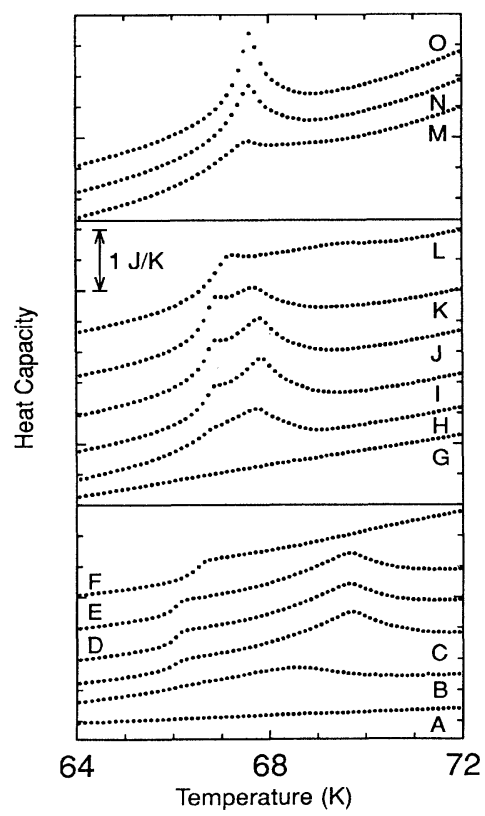

FIG. 2. Heat-capacity scans taken around the end points of the second-, third-, and fourth-layer layering transitions. Scans along the second and third layering transitions show two features each. Scans along the fourth layering transitions show only one peak. Coverages, in equivalent layers, are the following: $A, 1.20 ; B, 1.37 ; C, 1.63 ; D, 1.75 ; E, 1.82 ; F, 2.00 ; G, 2.60$; $H, 2.85 ; I, 3.00 ; J, 3.10 ; K, 3.15 ; L, 3.35 ; M, 4.65 ; N, 4.80$; and $O$, 4.95 .
We will consider the fourth-layer data in Sec. IIID. Scans $B-E$ and $H-K$ pass along the second and third layering transitions, respectively. Each of these scans shows two features. First, a steplike feature in the heat capacity occurs on the low-temperature side. In both layers two and three, there are three curves for which this step is seen at the same temperature to within experimental accuracy $(0.1 \mathrm{~K}), C, D$, and $E$, and $H, I$, and $J$. We interpret these features as two-dimensional triple points at $66.0 \pm 0.1 \mathrm{~K}$ for the second layer and $66.7 \pm 0.1 \mathrm{~K}$ for the third. We do not draw conclusions about the order of the melting transition at the triple point. A study of monolayer argon ${ }^{1}$ has concluded that melting at the 2D triple point is weakly first order because of very small but sharp peaks in the heat capacity that we do not detect in the second or third layers. It is possible that a very small sharp peak might not be observed in a scanning measurement.

The second feature seen is a cusplike anomaly at slightly higher temperature for those scans that pass through a triple point. We interpret this feature to be due to crossing the boundary between liquid-gas coexistence and uniform fluid. A feature in the heat capacity as this boundary is crossed has been shown to occur in other systems. ${ }^{35,36}$ The feature is partly due to the disappearance of one of the two phases, which causes a discontinuity in the slope of the specific heat and a step due to the disappearance of a term proportional to the latent heat of the liquid. Another contribution to the heat capacity is expected if the experimental path passes close to the twodimensional critical point. Around the critical point one expects a maximum in the heat capacity due to criticalpoint fluctuations. This maximum would not necessarily occur exactly as the experimental curve crosses the phase boundary. We believe that the measured heat capacities are due to a combination of these effects as well as a certain amount of broadening due to substrate inhomogeneity. Our interpretation of these peaks is strengthened by the scans at 1.63 and 1.82 layers (Fig. 2, $D$ and $E$ ) that show the cusp occurring at a lower temperature for a higher coverage scan. Thus, our data are consistent with monolayer studies and some multilayer studies showing rounded coexistence regions in various systems. Our best estimates of the layering critical-point temperatures, taken from the highest temperature cusp in each group, are $T_{c}(2)=69.7 \pm 0.1 \mathrm{~K}$ and $T_{c}(3)=67.8 \pm 0.1 \mathrm{~K}$. A measurement of the critical-point exponent $\beta$ governing the shape of the coexistence region near the critical point for layers two and three could be undertaken using slower scan rates for increased temperature resolution. This measurement has been performed in monolayer methane $^{35}$ and bilayer ethane ${ }^{36}$ yielding exponents close to Onsager's result of $\frac{1}{8}$ for the two-dimensional Ising model. ${ }^{37}$

The case for cusped anomalies occurring in the heat capacity at the coexistence boundary is further strengthened by the results of pressure measurements. Figure 3 shows the experimental trajectories plotted in $\left(\mu_{0}-\mu\right)^{(-1 / 3)}$ versus temperature for the low-temperature region, where $\mu$ is the chemical potential of the adsorbed system, and $\mu_{0}$ is the chemical potential of bulk matter at 


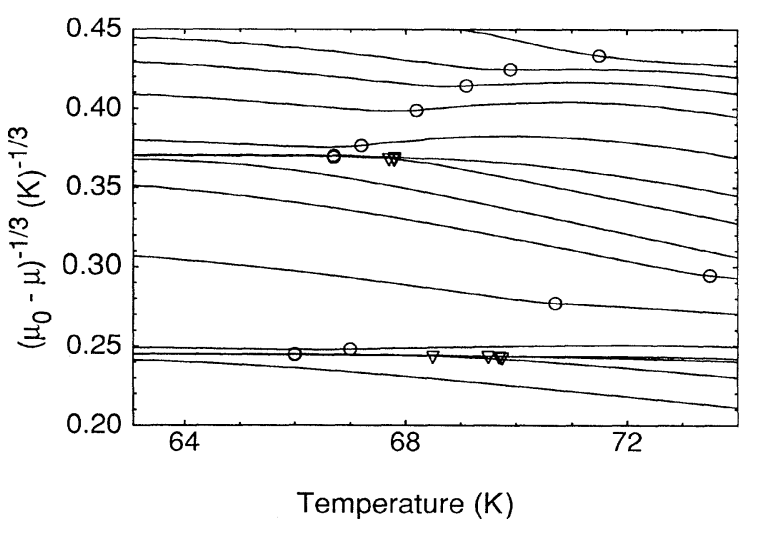

FIG. 3. Experimental trajectories in $\left(\mu_{0}-\mu\right)^{(-1 / 3)}$. Circles show the locations of melting transitions identified in the heatcapacity data. Triangles show the temperature corresponding to the cusp in the heat capacity. Chemical potentials are expressed in kelvins.

the same temperature. This function is chosen because $\left(\mu_{0}-\mu\right)^{(-1 / 3)}$ is proportional to the film thickness in the Frenkel-Halsey-Hill approximation. Curves at different film thicknesses that collapse in chemical potential indicate scans passing through coexistence regions and correspond to vertical steps in isotherms. Plotted in Fig. 3 are the locations of the heat-capacity steps and cusps. It is seen that cusp features occur just where the system departs from the coexistence region. Also, curves for which the step feature occurs above the triple-point temperature no longer pass through the liquid-gas coexistence region.

\section{Melting above the layer triple points, and registry transitions}

The triple-point temperature of the first layer of argon on graphite was found to be $47.2 \mathrm{~K}$ on the basis of the observation of small, sharp, coverage-independent peaks in a heat-capacity study. ${ }^{1}$ There is still some controversy over whether these peaks were correctly identified as melting. The small peak was followed by a much broader peak, which was tentatively identified as a transition from a substrate-induced hexatic phase to a uniform fluid phase. The small sharp peak was labeled as "weakly first order," so the melting process was not believed to occur by the KTHNY mechanism. Other studies (for a review, see Ref. 38) have concluded that the melting transition in monolayer argon is probably continuous. The triplepoint temperature of monolayer argon is below the temperature range of our experiment, but we are able to observe the melting of monolayer argon at much higher temperatures when more than one complete layer is adsorbed. We call this the extended monolayer regime. In this regime the first-order nature of the phase transition seems to be less ambiguous.

The tall sharp peaks in Fig. 4 are identified as firstlayer melting in the extended monolayer. At 1.00 layer, the coverage of the bottommost scan in Fig. 4, the first layer already melts at above $T_{t}$. The heat capacity at melting shows a sharp rise followed by a more gradual

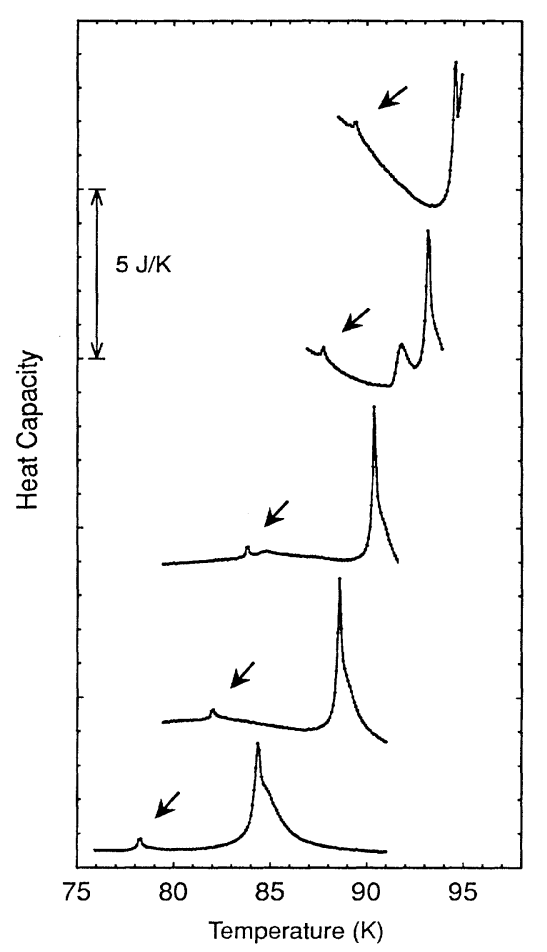

FIG. 4. Heat-capacity scans showing first-layer melting in the extended monolayer region. The small peaks, shown with arrows, are due to the proposed monolayer CI transition. Coverages at the melting peak are $1.00,1.14,1.31,1.59$, and 1.87 layers.

falloff. We believe that this behavior is the signature of a first-order transition. The width of the peak is due to crossing a narrow solid-liquid coexisting region. The peak is asymmetric instead of strictly mesalike probably due to the path the system takes through coexistence. The first-layer melting temperature increases to well over $95 \mathrm{~K}$ as the overlying film thickness increases.

One possible reason for a transition from continuous to first-order melting was suggested by Chan. ${ }^{38}$ It is thought that the continuous melting behavior in the monolayer occurs because of the strong orientational effect of the substrate. The lattice constant of monolayer argon is about $4.0 \AA{ }^{6}$ only slightly less than the commensurate value of $4.26 \AA$. As the monolayer is compressed by an overlying film, the orientational effect is expected to decrease, possibly resulting in a change in the melting behavior.

We cannot confirm such a picture from our data due to an additional complication. Small sharp peaks (arrows, Fig. 4) are observed in each scan approximately $6 \mathrm{~K}$ below the first-layer melting peaks. These peaks closely resemble peaks in the methane and/or graphite system where it is well established that they represent commensurate-incommensurate (CI) transitions, ${ }^{39,40}$ meaning a transition from a high-density (HD) phase at low temperature to a commensurate $(C)$ phase at higher temperature. In the case of methane and/or graphite, the existence of a $\mathbf{C}$ phase is confirmed by diffraction measurements (see references in Ref. 40 ), but the $C$ phase we 
postulate here for argon and/or graphite has not yet been detected in microscopic measurements. A possible alternative explanation of the peaks, that they are due a transition from HD to LD (low-density solid, meaning lower density than commensurate) is not tenable without an intervening commensurate phase, because there would be no change in either density or symmetry, hence no phase transition. We conclude, therefore, that the case for a CI transition is very strong. If this interpretation of the small peak is correct, then the first layer melts from a registered phase (just as methane does at high coverages $^{39}$ ). In a registered phase, Chan's argument given in the previous paragraph should not apply.

A tall rounded peak is seen between the first-layer CI transition and first-layer melting in the two highest coverage scans in Fig. 4. This peak actually crosses the first layer melting peak at higher coverages, so we do not believe that it is a phase transition in the film. Similar peaks were observed in methane on graphite and are believed to be due to the experimental path passing through a region of rapid desoprtion. ${ }^{30}$

The second-layer melting transition is the broad asymmetric mesa joining onto the second-layer triple point at low temperature (Fig. 5). We believe that melting occurs

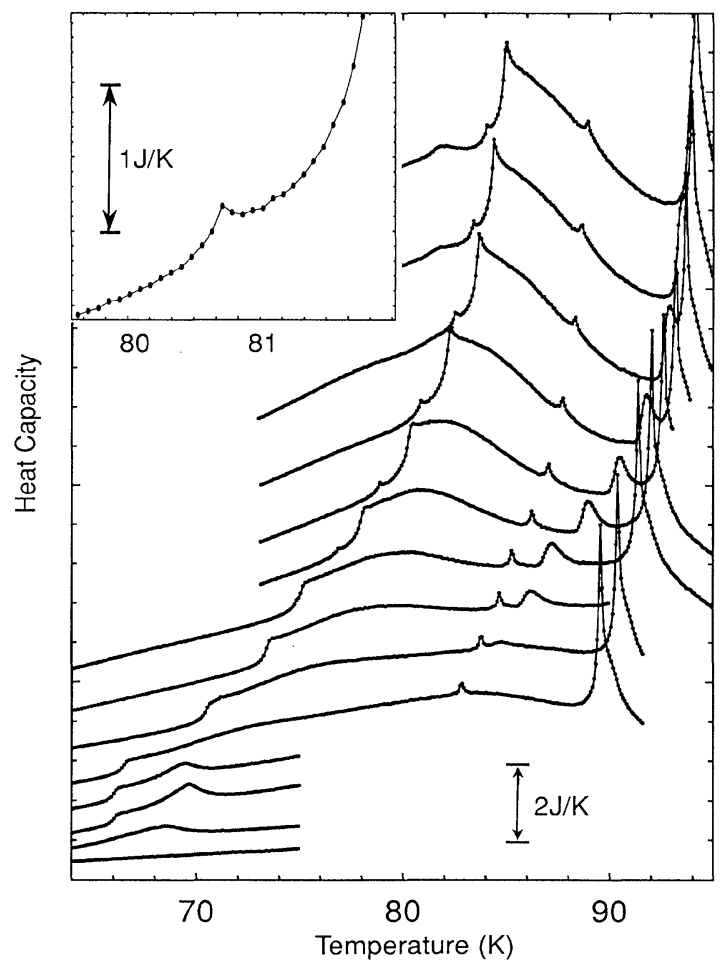

FIG. 5. Heat-capacity scans showing second-layer melting (large mesalike peaks) from around the layer triple point to coverages approaching three layers. First-layer melting and CI peaks are also shown at the high-temperature end of each scan. Coverages, at the second-layer melting peak, are 1.34, 1.60, 1.72, $1.79,1.96,2.14,2.26,2.34,2.42,2.50,2.57,2.64,2.70$, and 2.74 layers. The inset shows the second-layer premelting transition for the scan at 2.57 layers. at the sharp rise on the low-temperature side of the mesa. The pressure data show that mesas do not indicate a passage through a coexistence region. The large, sharp peaks at high coverages and/or temperatures indicate that the transition may be first order, but this conclusion is open to interpretation. It is possible that first-order behavior may evolve from continuous behavior as the coverage is increased. The origin of the broad mesa itself is not understood.

As the third layer begins to fill, we notice a very small peak (Fig. 5, inset) that precedes second-layer melting by about $1 \mathrm{~K}$ and continues up to high coverage. We believe that this peak indicates a CI transition occurring in the second layer. At low temperature, the second layer should be commensurate with the HD first layer, so it seems likely that, unlike in the first layer, the CI transition is from commensurate at low temperature to incommensurate (LD) at high temperature. Computer simulations $^{11}$ have shown that prior to a melting transition there is an increased upward mobility of atoms through the film that partially depopulates the melting layer. A decrease in density immediately before melting could be responsible for the CI transition we detect. This behavior might be detected in future simulations.

The third-layer melting transition is shown in Fig. 6. Above the maximum coverage of the third-layer liquidgas coexistence, the melting transition is manifested as a change in slope, accompanied by a small bump that may be a mesalike region similar to that seen in the second layer [Fig. 6(a)]. However, the mesalike features are much narrower than those due to second-layer melting, and they increase in temperature much less rapidly with coverage. Also, the mesas decrease in size with increasing coverage and change their shape entirely at a coverage of about 3.95 total layers adsorbed [Fig. 6(b)]. This behavior is consistent with the ellipsometry data of Youn and Hess ${ }^{10}$ who found reentrant first-order layering transitions for coverages above three layers. These are layering transitions that seem to reappear at temperatures above that of the layer critical point. Above the thirdlayer triple-point temperature, the mesalike features suggest that melting is first order. As the melting line increases in temperature and coverage, a more complicated phenomenon presumably occurs as it joins the reentrant layering transition, as we can no longer be sure of the order of the transition. These features seem to be related to a complicated series of phase transitions in the fourth and higher layers which we will describe in Sec. III D. We observe third-layer melting above $T_{t}$ [Fig. 6(c)] where it appears as a small rounded peak in contrast with the high-coverage second-layer melting peak (Fig. 5). Note that the coverages indicated for the high-coverage scans shown in Fig. 6 are to be regarded as the total amount adsorbed in the system including capillary condensation and the amount desorbed into the vapor. The rising heat-capacity peak near $T_{t}$ in each of the curves of Fig. 6(c) shows that a considerable amount of capillary condensation is beginning to occur. We are able to estimate that the film thickness is less than four layers as the third-layer melting transition passes through $T_{t}$.

Figure 7 is a proposed phase diagram based on the data 
for the first three layers. The positions of the heatcapacity peaks are plotted in temperature versus total number adsorbed in the film and capillary condensate. At low coverages, where little capillary condensate is present, the total number adsorbed can be taken to be approximately equal to the number in the film. However at higher coverages, around $3 \frac{1}{2}$ layers, the total number adsorbed is substantially greater than the number in the film. For example, the third-layer melting transition crosses $T_{t}$ at around $1000 \mathrm{~cm}^{3}$ at $\mathrm{STP}$ adsorbed. The chemical potential difference $\mu-\mu_{0}$ at this point is less than $\mu-\mu_{0}$ corresponding to fourth-layer condensation,
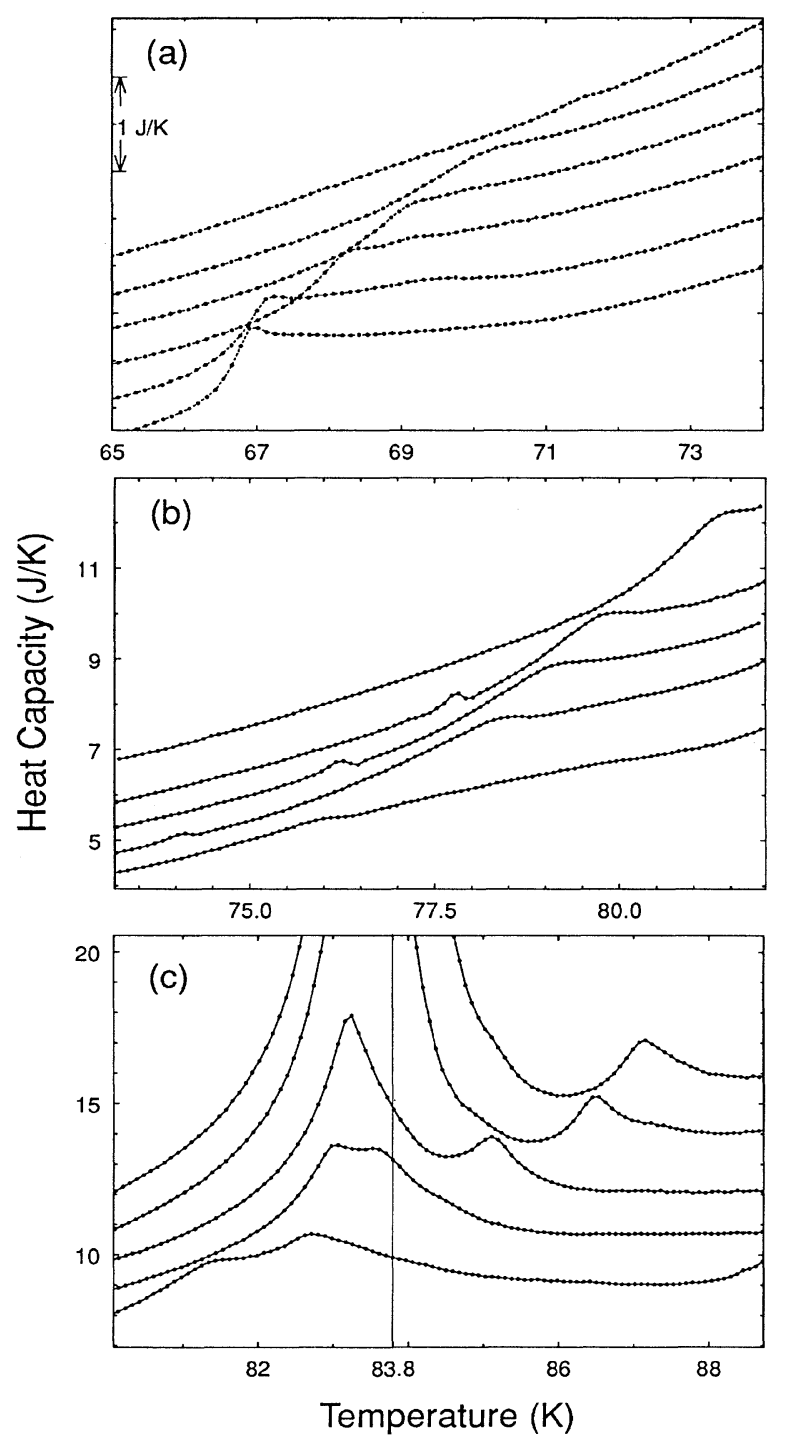

FIG. 6. (a) Heat-capacity scans showing third-layer melting at temperatures slightly above the layer triple point. Coverages are $3.15,3.28,3.50,3.63,3.75$, and 3.95 nominal layers. (b) Third-layer melting and possible reentrant layering transitions. Coverages are $3.95,4.18,4.20,4.23$, and 4.32 equivalent layers. (c) Third-layer melting as it passes through the bulk melting peak. Coverages are $4.32,4.61,5.26,6.63$, and 10.67 equivalent layers. Vertical line shows bulk $T_{t}$. so we expect the film thickness to be less than four layers. The regions in Fig. 7 are labeled as follows. DS, CS, ES, F, L, and $G$ represent dense solid, commensurate solid, expanded solid, fluid, liquid, and gas, respectively. $N$ DS means $N$ layers of solid believed to be mutually commensurate. The plus indicates a stratified film. For example, DS $+F$ means a dense solid first layer beneath a fluidlike second layer. The solidus indicates a coexistence region. For example, $\mathrm{DS}+(\mathrm{L} / \mathrm{G})$ means $\mathrm{L}$ and $\mathrm{G}$ phases coexisting in the second layer, on top of a DS first layer.

\section{Fourth, fifth, and sixth layers}

In contrast to the second and third layers, heatcapacity scans passing through the fourth-layer coexistence region only show one peak (Fig. 2, scans $M-O$ ). This behavior continues in the higher layers. The uppermost three curves in Fig. 2 show peaks associated with the fourth layer that occur at the same temperature $(67.5$ $\mathrm{K})$ and chemical potential to within experimental accuracy. These peaks mark the end of the first-order layering transition between three- and four-layer solid films. We will see later that at this point in the $(T, \mu)$ plane, the layering transition branches into two phase transitions the order of which we cannot determine. Thus, the point could either represent a triple point or a multicritical point. In the former case, the coexistence region following the triple point could be too narrow to observe. If the latter possibility is correct, we are observing a phenomenon radically different from what has previously been seen in multilayer films.

Figure 8(a) shows heat-capacity scans for coverages between three and four layers. Also shown are the locations of the heat-capacity features plotted in temperature versus $\left(\mu_{0}-\mu\right)^{-1 / 3}$, where $\mu_{0}$ is the chemical potential at the same temperature at bulk coexistence. This kind of plot gives approximately equal space on the ordinate to each layer of film, regardless of how much matter is capillary condensed. We argued in Sec. III C that the peaks seen at the lower coverages between 65 and $70 \mathrm{~K}$ in Fig. 8(a) are due to third-layer melting. At about $71 \mathrm{~K}$, corresponding to a nominal coverage of 4.15 , the peaks change form. In this region, measurements of $\mu$ versus $T$ (not shown), taken simultaneously with the heat-capacity scans, indicate that the scans pass through a coexistence region. The location of coexistence regions is shown with double lines in the $\left(\mu_{0}-\mu\right)^{-1 / 3}$ versus $T$ plot. The chemical potential at coexistence is nearly independent of temperature and is the same as $\mathrm{YH}$ find for the fourth-layer reentrant coexistence region. The heat capacity shows a small peak [arrows, Fig. 8(a)] as the system enters the coexistence region. A much broader feature follows that resembles the heat-capacity signature of the second- and third-layer liquid-gas coexistence regions. This feature is due to traversing the reentrant coexistence region. The pressure data show that each scan leaves the coexistence region just where the peak occurs at the end of this broad feature. For coverages higher than about 4.8 [Fig. 6(c)] the scans again show only one peak for third-layer melting, which continues upward in temperature to above the bulk triple point $(83.8 \mathrm{~K})$. Figure $8(\mathrm{~b})$ shows analogous 


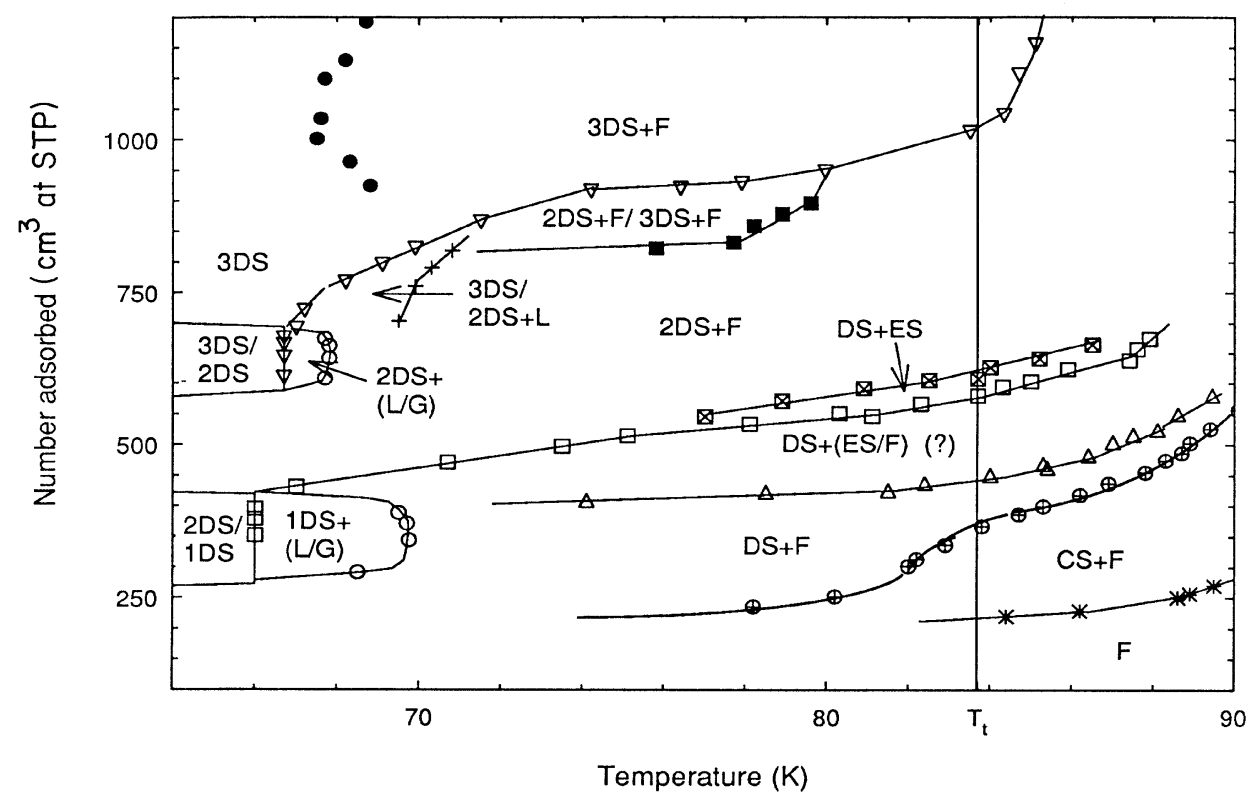

FIG. 7. Phase diagram for the first three layers in temperature vs number adsorbed in $\mathrm{cm}^{3}$ at STP (standard temperature and pressure). Notations are defined in the text. data for the fourth and fifth layers. We return to it below, after describing the overall phase diagram.

Figure 9 is a phase diagram based on our work and the results of the ellipsometry study of YH. The transitions marked by double lines in Fig. 9 are first-order transitions between phases of sufficient difference in density to produce a step noticeable in an adsorption isotherm or ellipsometry experiment. Up to the fourth layer, these regions are observable in our pressure measurements. For the locations of these transitions in higher layers, we rely on the data of $\mathrm{YH}$. The layering transitions at low temperature are between $N$ - and $N+1$-layer solid films. The phases in between are labeled $N S+\mathrm{G}$ to indicate that when the $N$ th layer becomes filled at nonzero temperature $(N S)$, it will typically adsorb some $2 \mathrm{D}$ gas $(\mathrm{G})$ before the solid $(N+1)$ st layer begins to nucleate.

Phase boundaries marked with single solid lines are believed to be melting transitions. These transitions are not detectable in the ellipsometry data, but show up strongly in the heat-capacity data. We see that such transitions join the $N$ th-layering transition with the reentrant layering transition approximately half a layer above. As we saw in the second and third layers, only the topmost layer appears to melt when passing through one of these transitions. Accordingly, we label the phases between the reentrant transitions as $N S+\mathrm{F}$ by which we mean $N$ layers of solid film underneath one disordered (fluid) layer.

Also noticeable are heat-capacity peaks that join the layering transitions with the reentrant transitions approximately half a layer below. It is possible that these peaks are due to phase transitions, shown with dashed lines in Fig. 9, that distinguish the $N S+\mathrm{G}$ phase from the $N S+F$ phase. If these are phase transitions, they should be first order because the phases they separate do not differ in symmetry, and hence, must differ in density. It is also possible that these peaks do not represent true phase transitions, but a region in which the density of the uppermost layer changes rapidly and continuously.
Heat-capacity features due to the reentrant region between $3 S+F$ and $4 S+F$ are shown in Fig. 8(b). These features are much smaller than those shown in Fig. 8(a), and there is no longer a separate feature caused by entering the coexistence region. Heat-capacity features due to the coexistence region between $4 S+\mathrm{F}$ and $5 S+\mathrm{F}$ (not shown) are barely noticeable against the background signal (due mainly to desorption of the film). We do not observe any heat-capacity signals due to reentrant layering transitions in higher layers. Thus we see that as we move upwards in coverage, the heat-capacity signature of the reentrant regions disappears.

Heat-capacity peaks, between 65 and $70 \mathrm{~K}$, associated with the fifth and sixth layers, repeat the trend set in the fourth layer. Figure 10 shows the peaks as they zigzag back and forth between layering and reentrant layering transitions. The amplitude of the zigzags decreases going upwards in coverage, implying that the upper bound of the low-temperature layering transitions and the lower bound of the reentrant transitions approach a common temperature in the limit of an infinitely thick film.

The phase diagram of Fig. 9 could be the signal of a PR transition at a bulk surface. PR would occur around 68.5 K, which is where the end points of the lowtemperature layering transitions seem to converge. The DOF phase would occur in the region in which reentrant layering transitions between films with disordered surfaces are seen. The existence of these reentrant layering transitions indicates that the crystal surface resists the formation of unlimited steps in this range of temperatures. YH calculates that the occupancy of the surface layer in the reentrant layering region is close to $\frac{1}{2}$ (they estimate 0.7 ). In the treatment by den Nijs of the RSOS model the occupancy in the DOF phase is expected to be $\frac{1}{2}$ due to the symmetry between up and down steps. Our data are consistent with these estimates. Thus we see that the reentrant regions embody the main characteristics of the DOF phase. In this interpretation, roughening 

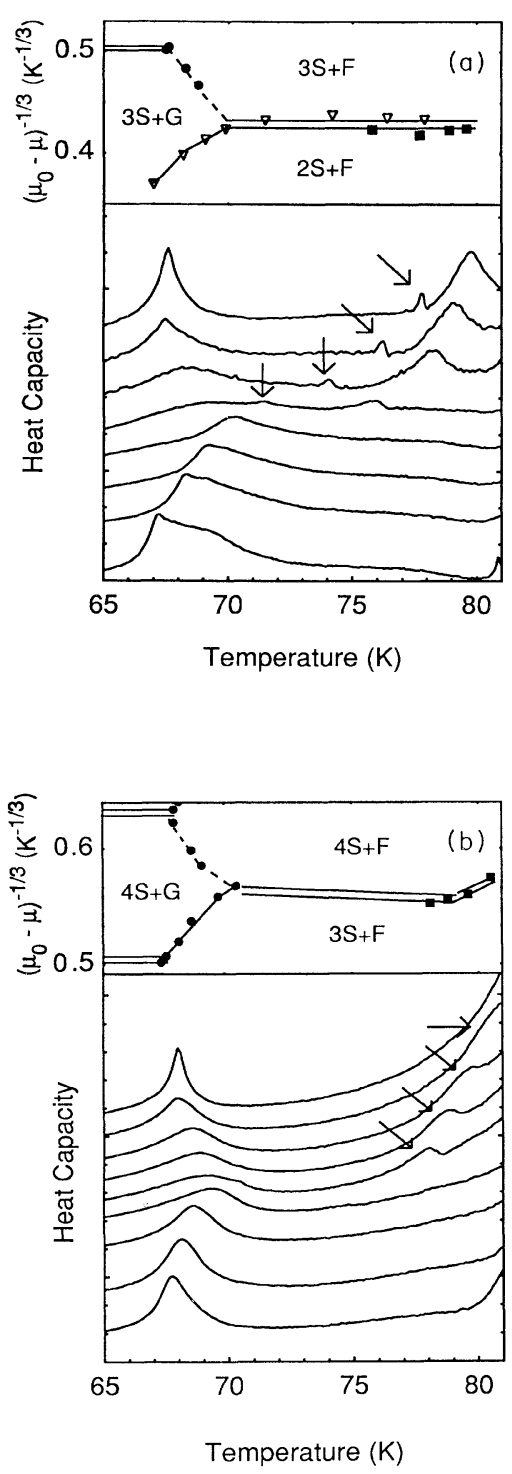

FIG. 8. (a) (Bottom) Heat-capacity scans between three and four layers corrected for desorption. The lowest curves have features at low temperature due to third-layer melting. After about $71 \mathrm{~K}$ two small peaks are seen where each of the four uppermost scans enters and leaves the reentrant coexistence region. The coverages (at $65 \mathrm{~K}$ ) are $3.31,3.54,3.70,3.84,4.09$, 4.44, 4.59, and 4.74 nominal layers. (Top) Positions of heatcapacity peaks plotted in temperature vs inverse cube root chemical potential difference (in $\mathrm{K}^{-1 / 3}$ ). Open triangles are third-layer melting and the small peak seen entering the thirdlayer reentrant layering transition. Filled squares show where experimental paths are observed to leave a reentrant coexistence region. Filled circles are the locations of the low-temperature peaks for layers four and up. (b) (Bottom) Heat-capacity scans between four and five layers corrected for desorption. The shallow peaks around $79 \mathrm{~K}$ are caused by the system leaving the coexistence region. The nominal coverages (at $65 \mathrm{~K}$ ) are 5.04, $5.19,5.49,5.83,6.11,6.48,6.797 .24$, and 7.65 layers. (Top) Positions of heat-capacity peaks plotted in temperature vs inverse cube root chemical potential difference (in $\mathrm{K}^{-1 / 3}$ ).

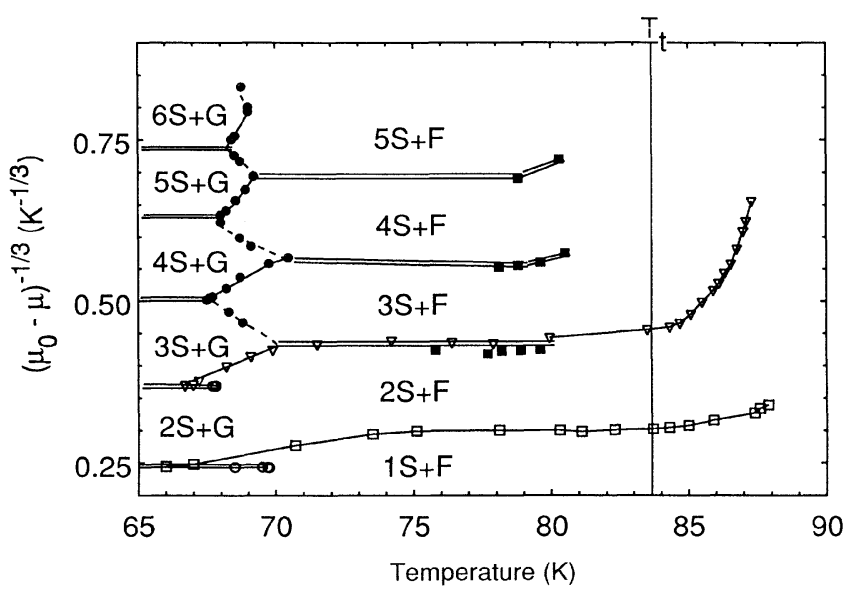

FIG. 9. Phase diagram in temperature vs inverse cube root chemical potential difference (in $\mathrm{K}^{-1 / 3}$ ). Open circles indicate 2D liquid-gas coexistence in layers two and three. Open squares are second-layer melting. Open triangles are third-layer melting. Filled circles are the locations of the low-temperature peaks for layers four and up. Filled squares show where experimental paths are observed to leave a reentrant coexistence region.

would occur at about $80 \mathrm{~K}$, the high-temperature end of the reentrant layering transitions.

In our data the heat-capacity signature of the reentrant layering transitions disappears as the film becomes thicker, but the peaks due to the low-temperature layering transitions have about the same latent heat regardless of coverage. This behavior is reasonable because the lowtemperature transitions always represent the disordering of one layer whereas the reentrant transitions represent only the disappearance of the difference between $N S+F$ and $(N+1) S+F$ films, which becomes unimportant energetically as the film thickness increases. In the bulk,

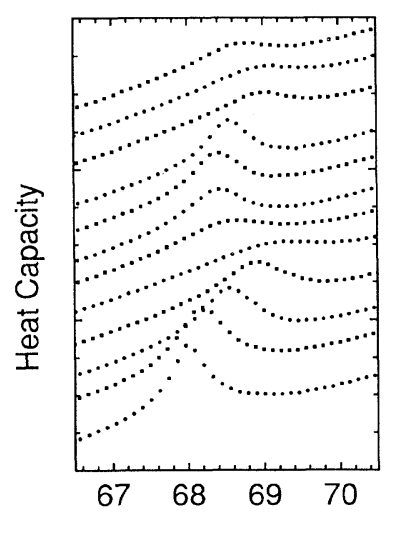

Temperature $(\mathrm{K})$

FIG. 10. Heat-capacity peaks seen in layers five and six. These peaks zigzag between the low-temperature and reentrant layering transitions. The heat capacities are not corrected for desorption. 
roughening is expected to be an infinite-order phase transition, unobservable in heat-capacity measurements. If the interpretation of our data in terms of preroughening is correct, the heat-capacity peaks for the thicker films shown in Fig. 10 represent a direct observation of a PR transition.

\section{CONCLUSIONS}

Until quite recently, a simple and appealing picture seemed to have emerged of how thin films of simple substances such as argon may evolve into bulk matter. However, evidence has recently begun to call this picture into question. In this and a number of companion studies, ${ }^{32,39}$ using calorimetry much more sensitive than was previously available, combined with simultaneous vapor pressure measurements, and armed with a better understanding of how to take capillary condensation in to account, we have confirmed that the truth is considerably more complex and more interesting than was previously suspected.

According to the previous view, the first layer has a rich two-dimensional phase diagram including gas, liquid, and solid phases. In some substances, e.g., methane, the solid phase could be commensurate with the substrate, or incommensurate at lower density or higher density, depending on temperature and coverage. In argon, on the other hand, no commensurate phase had been detected at all. Thicker films could be understood in terms of a simple lattice-gas model, ${ }^{41}$ the completed first layer forming the underlying lattice. Each layer would undergo an Isinglike critical point, $T_{c}(N)$, that terminates a first-order layering transition. As the layer number $N$ increases to infinity, $T_{c}(N)$ would tend to the roughening temperature of the bulk interface, $T_{R}$. At somewhat higher temperatures, but still below the bulk triple point, $T_{t}$, a separate phenomenon, called surface melting in the bulk, or stratified melting in the film, would occur. ${ }^{42}$ Surface melting could be understood to be a consequence of the fact that, if the liquid phase wet the solid-vapor interface, the system could lower its free energy at $T<T_{t}$ by interspersing a film of liquid between the solid and the vapor. Bulk melting at $T_{t}$ could then be understood as the propagation of the liquid-solid interface from near the vapor interface, into the bulk. It was also noticed that the reverse phenomenon could occur: if the solid phase wet the liquid-substrate interface, a solid film at the interface would be expected at $T>T_{t}$. This phenomenon was termed substrate-induced freezing. ${ }^{26}$

Our results confirm the earlier observation by Zhu and Dash $^{3}$ that a film of solid does, in fact, persist at the substrate interface for $T>T_{t}$. Indeed, the first three layers are each seen to melt at temperatures above $T_{t}$ that increase with increasing thickness of overlayers adsorbed on top of them. Qualitatively similar behavior is observed in methane, where the first layer, and possibly the second, are seen to melt at $T>T_{t}$. However, our data indicate that the first layer probably forms a commensurate phase just before it melts (the same occurs in methane), and the second layer appears to form an incommensurate (with respect to the first layer) film just before it melts. The third layer undergoes a broad two-phase melting region between 70 and $80 \mathrm{~K}$ that seems to be a precursor of an entirely new kind of behavior in the fourth and higher layers, known as reentrant layering. ${ }^{10}$

If surface melting occurs in argon, we find no evidence for it in our measurements. We do find that observations previously taken to be evidence of surface melting may instead be understood as being due to the twodimensional melting of the uppermost layer, or to the melting of capillary condensate.

Our findings may be summarized as follows: the second and third layers of argon adsorbed on graphite each have a complex two-dimensional phase diagram much like the first layer. The solid first layer forms an inert substrate for the second layer, and the second does likewise for the third. At layers higher than the third, the system goes over from layer-by-layer formation of two-dimensional matter into an approach to formation of the bulk interface.

\section{ACKNOWLEDGMENTS}

We have enjoyed fruitful discussions with Dr. Marcel den Nijs and Dr. Peter Weichman. This work was supported by DOE Grant No. DE-FG03-85ER45192.

${ }^{1}$ A. D. Migone, Z. R. Li, and M. H. W. Chan, Phys. Rev. Lett. 53, 819 (1984).

${ }^{2}$ D. M. Zhu and J. G. Dash, Phys. Rev. Lett. 60, 432 (1988).

${ }^{3}$ D. M. Zhu and J. G. Dash, Phys. Rev. B 38, 11673 (1988).

${ }^{4}$ T. T. Chung, Surf. Sci. 87, 348 (1978).

${ }^{5}$ Y. Larher and B. Gilquin, Phys. Rev. A 20, 1599 (1979).

${ }^{6}$ J. P. McTague, J. Als-Nielson, J. Bohn, and M. Nielsen, Phys. Rev. B 25, 7765 (1982).

7J. Z. Larese and Q. M. Zhang, Phys. Rev. Lett. 64, 922 (1990).

${ }^{8}$ J. Z. Larese, Q. M. Zhang, L. Passell, and J. M. Hastings, Phys. Rev. B 40, 4271 (1989).

${ }^{9}$ J. M. Gay, J. Suzanne, J. G. Dash, and H. J. Lauter, J. Phys. (Paris) 1, 1279 (1991).

${ }^{10}$ H. S. Youn and G. B. Hess, Phys. Rev. Lett. 64, 918 (1990).

${ }^{11}$ J. M. Phillips, Phys. Lett. A 147, 54 (1990).

${ }^{12}$ A. L. Cheng and W. A. Steele, Langmuir 5, 600 (1989).

${ }^{13}$ G. D. Halsey, J. Chem. Phys. 16, 931 (1948); T. L. Hill, ibid. 15, 767 (1947).

${ }^{14}$ G. B. Hess, in Phase Transitions in Surface Films 2, edited by H. Taub et al. (Plenum, New York, 1991).

${ }^{15}$ M. J. De Olivera and R. B. Griffiths, Surf. Sci. 71, 687 (1978).

${ }^{16}$ I. M. Kim and D. P. Landau, Surf. Sci. 110, 415 (1981).

${ }^{17}$ D. A. Huse, Phys. Rev. B 30, 1371 (1984).

${ }^{18}$ M. P. Nightingale, W. F. Saam, and M. Schick, Phys. Rev. B 30, 3830 (1984).

${ }^{19}$ M. P. Nightingale, W. F. Saam, and M. Schick, Phys. Rev. Lett. 51, 1275 (1983); W. F. Saam, Surf. Sci. 125, 253 (1983); J. D. Weeks, Phys. Rev. B 26, 3998 (1982); J. M. Luck, S. Liebler, and B. Derrida, J. Phys. (Paris) 44, 1135 (1983).

${ }^{20}$ M. H. W. Chan and Y. P. Feng, Bull. Am. Phys. Soc. 33, 283 (1988).

${ }^{21}$ A. J. Jin, M. R. Bjurstrom, and M. H. W. Chan, Phys. Rev. 
Lett. 62, 1372 (1989).

${ }^{22}$ H. Freimuth and H. Wiechert, Surf. Sci. 162, 432 (1985).

${ }^{23}$ A. D. Migone, J. G. Dash, M. Schick, and O. E. Vilches, Phys. Rev. B 34, 6322 (1986).

24J. Ma, T. A. Sullivan, and O. E. Vilches, J. Appl. Phys. 26-3, 263 (1987).

${ }^{25}$ D. M. Zhu, Ph.D. thesis, University of Washington, 1988.

${ }^{26}$ M. S. Pettersen, M. J. Lysek, and D. L. Goodstein, Phys. Rev. B 40, 4938 (1989).

27J. D. Weeks, in Ordering in Strongly Fluctuating Condensed Matter Systems, edited by T. Riste (Plenum, New York, 1979), p. 293.

${ }^{28}$ K. Rommelse and M. den Nijs, Phys. Rev. Lett. 59, 2578 (1987); M. den Nijs, ibid. 64, 435 (1990); M. den Nijs and K. Rommelse, Phys. Rev. B 40, 4709 (1989).

${ }^{29}$ F. Ser, Y. Larher, and B. Gilquin, Mol. Phys. 67, 1077 (1989).

${ }^{30}$ M. J. Lysek, Ph.D. thesis, California Institute of Technology, 1991.

${ }^{31}$ M. J. Lysek, P. K. Day, M. LaMadrid, and D. L. Goodstein, Rev. Sci. Instrum. 63, 5750 (1992).

${ }^{32}$ M. J. Lysek, M. LaMadrid, P. K. Day, and D. L. Goodstein, Langmuir 8, 898 (1992).

${ }^{33}$ The temperature of the cell was controlled during the cooling cycle so that the cooling rate satisifed $|d T / d t|<C\left(t_{1}-t\right)^{3}$.
Any cooling schedule that slows the cooling rate at low temperature where diffusion times are large should aid in the formation of uniform films. The total cooling time from 90 to 62 $\mathrm{K}$ was $12 \mathrm{~h}$.

${ }^{34}$ E. Cheng and M. W. Cole, Phys. Rev. B 41, 9650 (1990).

${ }^{35}$ H. K. Kim and M. H. W. Chan, Phys. Rev. Lett. 53, 170 (1984).

${ }^{36}$ H. K. Kim, Y. P. Feng, Q. M. Zhang, and M. H. W. Chan, Phys. Rev. B 37, 3511 (1988); Q. M. Zhang, Y. P. Feng, H. K. Kim, and M. H. W. Chan, Phys. Rev. Lett. 57, 1456 (1986).

${ }^{37}$ L. Onsager, Phys. Rev. 65, 117 (1944); C. N. Yang, Phys. Rev. 85, 808 (1952).

${ }^{38}$ M. H. W. Chan, in Phase transitions in Surface Films 2, edited by H. Taub et al. (Plenum, New York, 1992).

${ }^{39}$ M. J. Lysek, M. LaMadrid, P. K. Day, and D. L. Goodstein, Phys. Rev. B (to be published); P. K. Day, M. J. Lysek, M. LaMadrid, and D. L. Goldstein, Phys. Rev. B (to be published).

${ }^{40}$ H. K. Kim, Q. M. Zhang, and M. H. W. Chan, Phys. Rev. B 34, 4699 (1986).

${ }^{41}$ R. Pandit, M. Shick, and M. Wortis, Phys. Rev. B 26, 5112 (1982).

42J. G. Dash, Contemp. Phys. 30, 89 (1989). 